Scientific Visualization, 2019, volume 11, number 5, pages 119 - 125, DOI: 10.26583/sv.11.5.10

\title{
Reconstruction of interference and Hilbert structures from numerical models of the isotherm field in convective flows induced in a vertical layer of water by unsteady boundary conditions
}

\author{
V.A. Arbuzov',A,C, E.V. Arbuzov²,B,C, V.S. Berdnikov3,A,C, \\ Yu.N. Dubnishchev4,A,C, S.A. Kislitsin ${ }^{5, A, C}$, O.S. Zolotukhina ${ }^{6, C}$ \\ A Kutateladze Institute of Thermophysics Siberian Branch of the \\ Russian Academy of Sciences, Novosibirsk, Russia \\ B Sobolev Institute of Mathematics Siberian Branch of the \\ Russian Academy of Sciences, Novosibirsk, Russia \\ c Novosibirsk State Technical University, Novosibirsk, Russia \\ ${ }^{1}$ ORCID: 0000-0001-7874-039X, dubnistchev@itp.nsc.ru \\ 2 ORCID: 0000-0003-2404-326X \\ 3 ORCID: 0000-0001-9488-8650 \\ 4 ORCID: 0000-0002-7819-1340 \\ 5 ORCID: 0000-0002-4260-7025 \\ 6 ORCID: 0000-0003-3486-4459
}

\begin{abstract}
The problem of reconstructing interference and Hilbert structures from a numerical model of the evolution of the thermal field of convective flows in a vertical water layer bounded by flat heat-exchanging surfaces under unsteady boundary conditions in the monotonic cooling mode and taking into account density inversion at a temperature of +4 degrees Celsius was solved. The simulation of the thermal fields of convective flows in the form of the dynamic structure of isotherms was carried out taking into account the nonlinear dependence of thermal conductivity and water density on temperature. The field of the phase function, its Hilbert image and the interference field, which are compared with the results of the interference and Hilbert visualization of the fields of phase optical density obtained in the experiment, were reconstructed from the isotherms field supplemented by calculating of the velocity field and of the temperature gradients field. The presented films illustrate the qualitative adequacy of the coevolution of numerical models and real processes.
\end{abstract} currents.

Keywords: optical diagnostics, Hilbert-optics, shear interferometry, convective

\section{Introduction}

Interest in the study of convective flows is associated with the special importance of convection in geodynamics, physics of the atmosphere and ocean, in hydrodynamic and thermophysical processes associated with the formation and growth of crystals [1]. The relevance of this kind of research has recently increased due to the observed anomalies in the formation and melting of ice in the Arctic and Antarctic regions, as well as the intensification of the development of offshore oil and gas-bearing areas of the Arctic. Convective structures in a vertical water layer bounded by flat heat exchange surfaces under unsteady boundary conditions are visualized in [2] by methods of Hilbert optics and shear interferometry. Numerical simulation of temperature field as of field of isotherms in the mode of monotonic 
cooling of vertical walls is performed. The inverse problem of reconstruction of interferograms and hilbertograms from the numerical model of the temperature field is solved fragmentally.

In the course of the development of these studies, the problem arises of reproducing the coevolution of reconstructed interference fields and Hilbert structures of convective flows in comparison with the results obtained in experiments. The novelty of the problem is due to the almost complete lack of data on the development of convective flows in the conditions of monotonic temperature changes on the vertical walls, taking into account the inversion of the water density in the vicinity of the isotherm $\left(+4^{\circ} \mathrm{C}\right)$ and consists in a comprehensive approach combining the results of a physical and numerical experiment in which the simulation of the isotherm field is supplemented by the calculation of the velocity field and temperature gradients.

\section{Method}

The description of the research complex containing the experimental stand and optical diagnostics system is given in [2]. The experimental stand is a rectangular cavity filled with water, with transparent optical-quality glasses. The internal dimensions of the cavity are $136 \times 86 \times 30 \mathrm{~mm}$. Side walls are formed by parallel arranged cooled flat copper plates. On the outer side of the plates there are cavities into which coolant is supplied from the cryostat and thermostat. The temperature of one of the plates by pumping coolant from the cryostat can reach minus $28^{\circ} \mathrm{C}$. The temperature of the other plate is maintained in the range from room temperature to $+8^{\circ} \mathrm{C}$. The optical diagnostics system by the methods of Hilbert optics and shear interferometry performs visualization of perturbations gradients of the phase optical density induced in the water layer by non-stationary temperature boundary conditions on vertical heat exchange surfaces limiting the medium under study.

Figure 1 shows a frame from the video-film attached to the article, which illustrates the results of visualization of the phase density field by Hilbert-optics $(a)$ and shear interferome$\operatorname{try}(b)$.

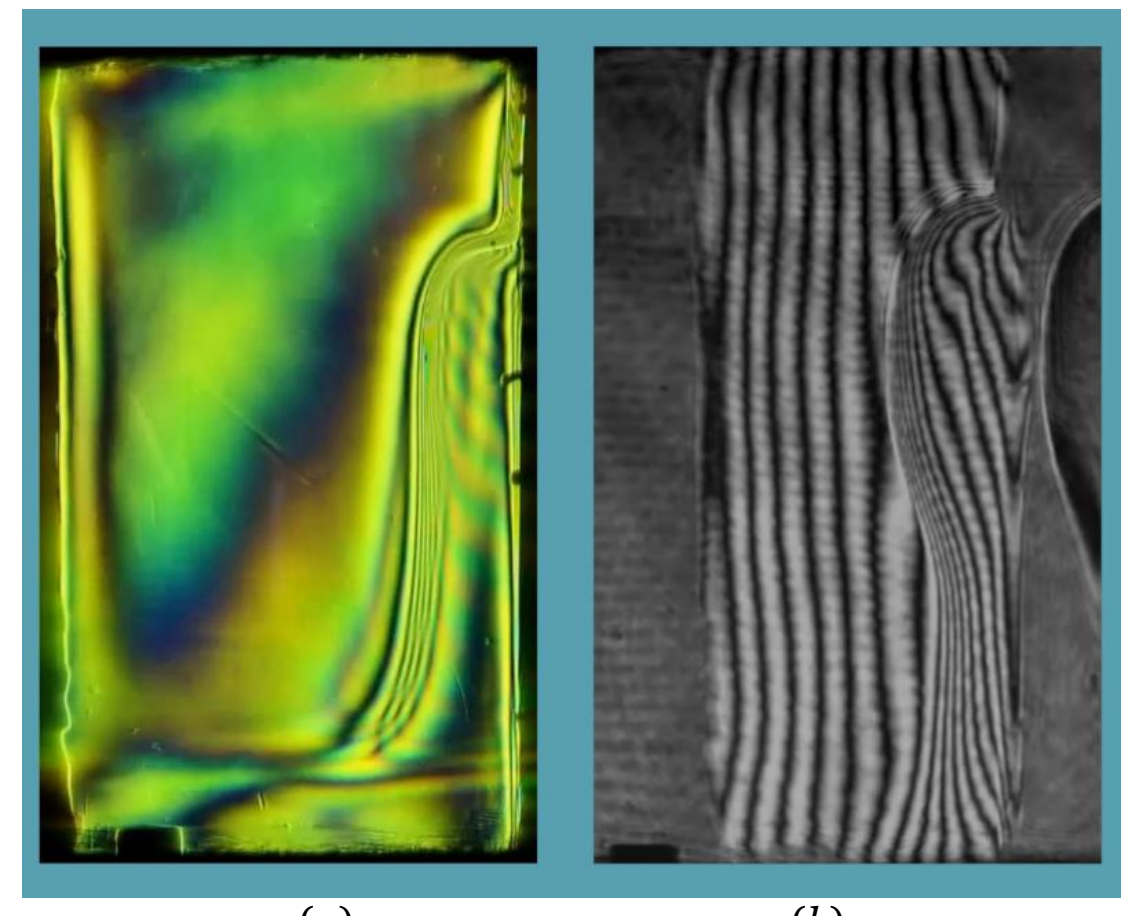

(a)

(b)

Fig. 1. Figure 1 shows a frame from the video-film attached to the article, which illustrates the results of visualization of the phase density field by Hilbert-optics (a) and shear interferometry (b). 
As a verification of the results obtained in [2], numerical simulation of the temperature fields of convective flows in the form of a dynamic structure of isotherms is performed. From these structures, shear interferograms and hilbertograms were reconstructed, which were compared with the results obtained in the experiment.

Convective heat transfer in a liquid in a two-dimensional formulation was described by a dimensionless system of Navier-Stokes equations, energy and continuity in the approximation Boussinesq. Conductive heat transfer in massive horizontal walls of Plexiglas was modeled using the heat transfer equation.

The equations were solved numerically by the finite element method in the conjugate formulation. Dependences of density and coefficient of volumetric thermal expansion on temperature were taken into account. At the solid boundaries for the velocity (and, accordingly, for the current function), the conditions of adhesion and non-flow through were assigned. The boundary condition for the vortex is obtained from the field of the function of current using the method of conjugate results [3]. At the interface of the liquid with solid walls the condition of continuity of temperature and heat flow was set.

An uneven triangular grid, thickened bunched to varying degrees to all the boundaries of the computational domain, with the number of nodes $\approx 4^{*} 10^{4}$ was used. Linear basis functions be given on the elements. A cellular step-by-step algorithm for the maximum angle was used to construct the triangulation [4]. The program implements an iterative process, in which, if necessary, the necessary values of the variables from the previous steps and the calculated values of the coefficients on the parameters are substituted.

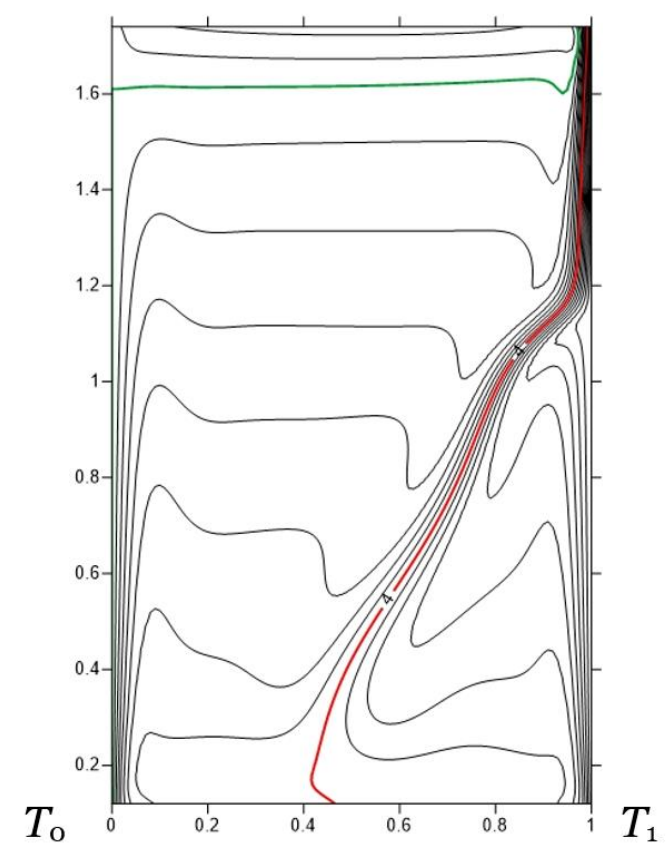

(a)

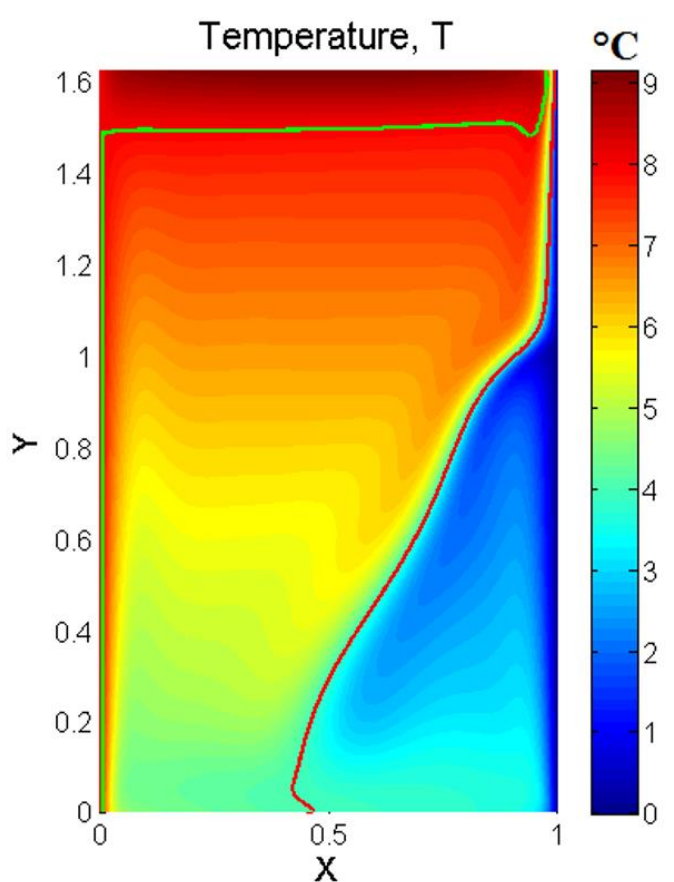

(b)

Fig. 2. Numerical model: (a) - isotherm field ( $\mathrm{To}=8^{\circ} \mathrm{C}, \mathrm{T} 1=\mathrm{O}^{\circ} \mathrm{C}$ );

(b) - numerical model of the temperature field from the initial field of isotherms

(3545 seconds from the moment the heat exchange surfaces are switched on, frame 901).

The problem is solved in a non-stationary situation: the initial temperature of the system is $+20^{\circ} \mathrm{C}$, the left vertical wall is monotonically cooled to $+8^{\circ} \mathrm{C}$ for 2500 seconds. The right wall was cooled to $\mathrm{O}^{\circ} \mathrm{C}$ for 2500 seconds. The outer surfaces of the horizontal walls are adiabatic. In this paper, the simulation of the isotherms field is supplemented by the calculation of the velocity field and temperature gradients.

Temperature fields are reconstructed from the numerically models of isotherms (figure $2, a$ ). The graphs are constructed in relative units, (in the figures the real size of the cuvette 
width equal to $86 \mathrm{~mm}$ is taken as a scale in the transition to dimensionless equations and boundary conditions).

Temperature boundary conditions on the left and right walls are indicated, respectively, $T_{1}$ and $T_{2}$. The visualized hilbertograms represent quasi-gradiented structure of the optical phase field $\Delta \varphi(x, y, T)$, induced in the under investigation environmental by the temperature boundary conditions:

$$
\Delta \varphi(x, y, T)=k\left[n(x, y, T)-n_{0}\right] l,
$$

where $l$ is the thickness of the liquid layer, $k=2 \pi / \lambda$ is the wave number of the light field, $n(x, y, T)$ is the refractive index of the liquid as a function of the spatial coordinates $x, y$ and temperature $T$.

This function is determined by the temperature dependence of the specific refraction of water. Temperature dependence of the refractive index of distilled water (Fig. 3) was calculated, according to $[5,6]$, on the density and specific refraction at a wavelength $\lambda=589.3 \mathrm{Nm}$ at normal atmospheric pressure.

The optical phase field was calculated from the spatial distribution of the refractive index (Fig. 4). The Hilbert transformation of this field was performed and the shear interferograms was reconstructed.

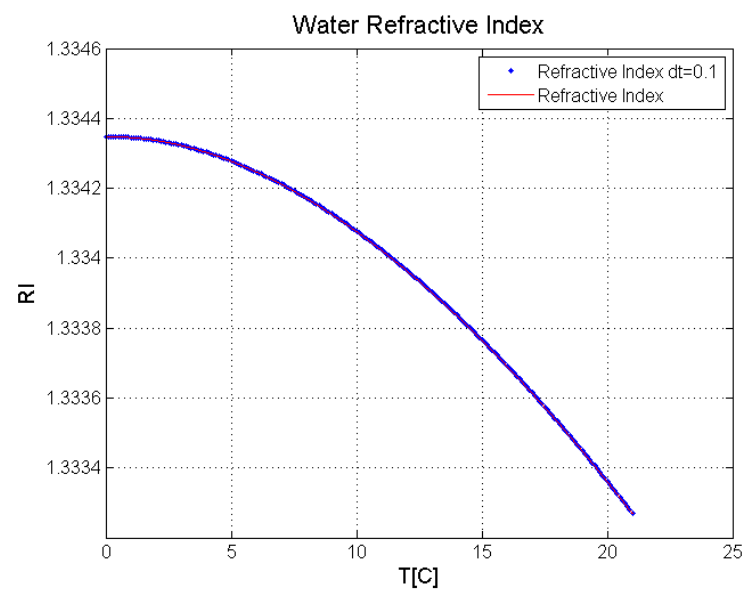

Fig. 3. The dependence of the refractive index of distilled water on temperature from $-2^{\circ} \mathrm{C}$ to $+21^{\circ} \mathrm{C}[5,6]$.

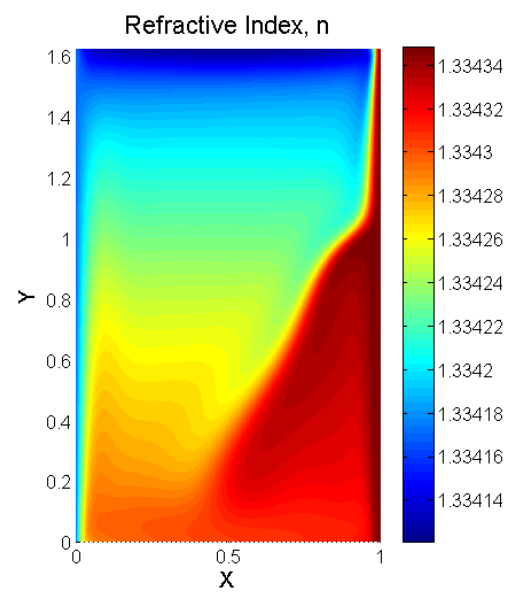

Fig. 4. Field of refractive index of distilled water ( $\mathrm{x}, \mathrm{y}$ axis).

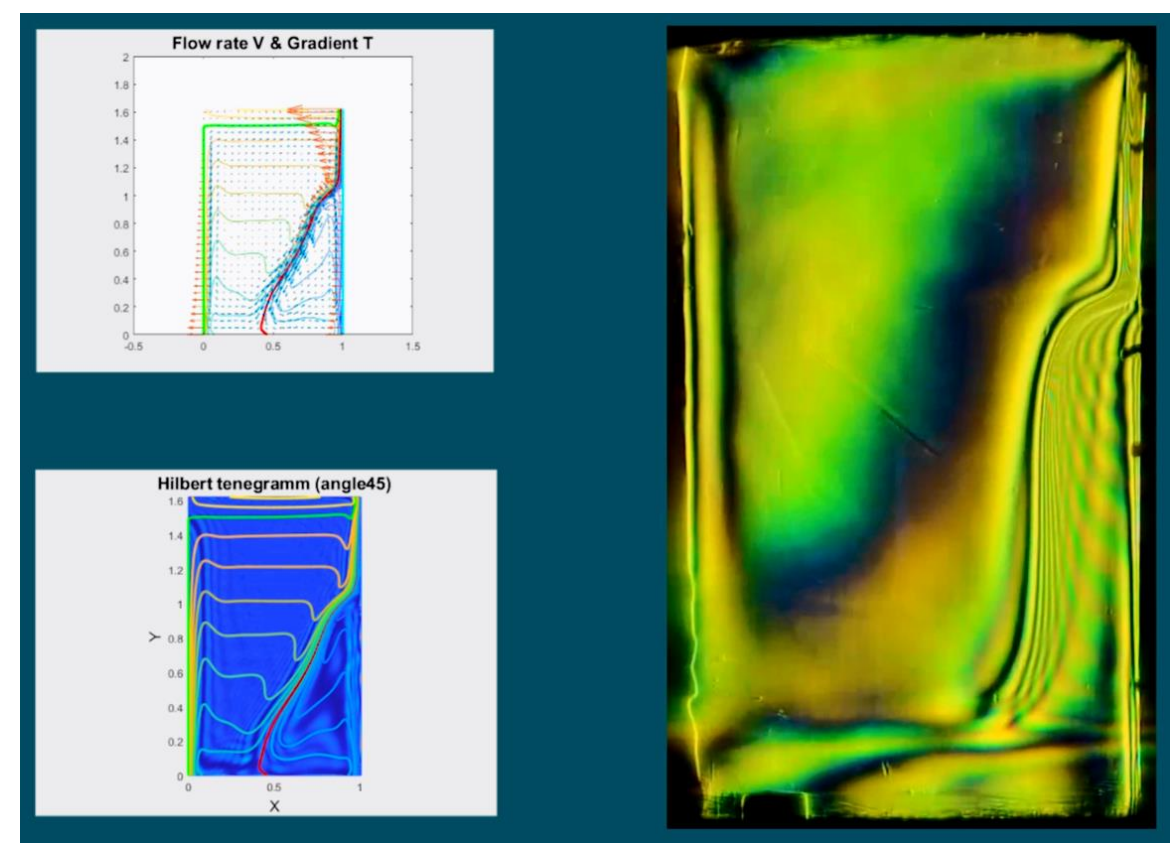

Fig. 5. Reconstruction of Hilbert fields of phase optical density. 
Figure 5 shows a frame from the video attached to the article, which shows the numerical fields of isotherms, temperature gradients and velocities (at the top left). At the bottom left is a Hilbert image of the phase density field (blue color) reconstructed from these numerically obtained isotherm fields. Isotherms corresponding to temperatures of $+4^{\circ} \mathrm{C}$ and $+8^{\circ} \mathrm{C}$ are highlighted in red and green.

On the right, the obtained Hilbert-visualized optical phase density field is shown, with which the evolution of the fields of isotherms and temperature gradients obtained numerically is synchronized. The film demonstrates the qualitative correspondence between the results of the experiment and simulation.

Figure 6 shows a frame from another video attached to the article.

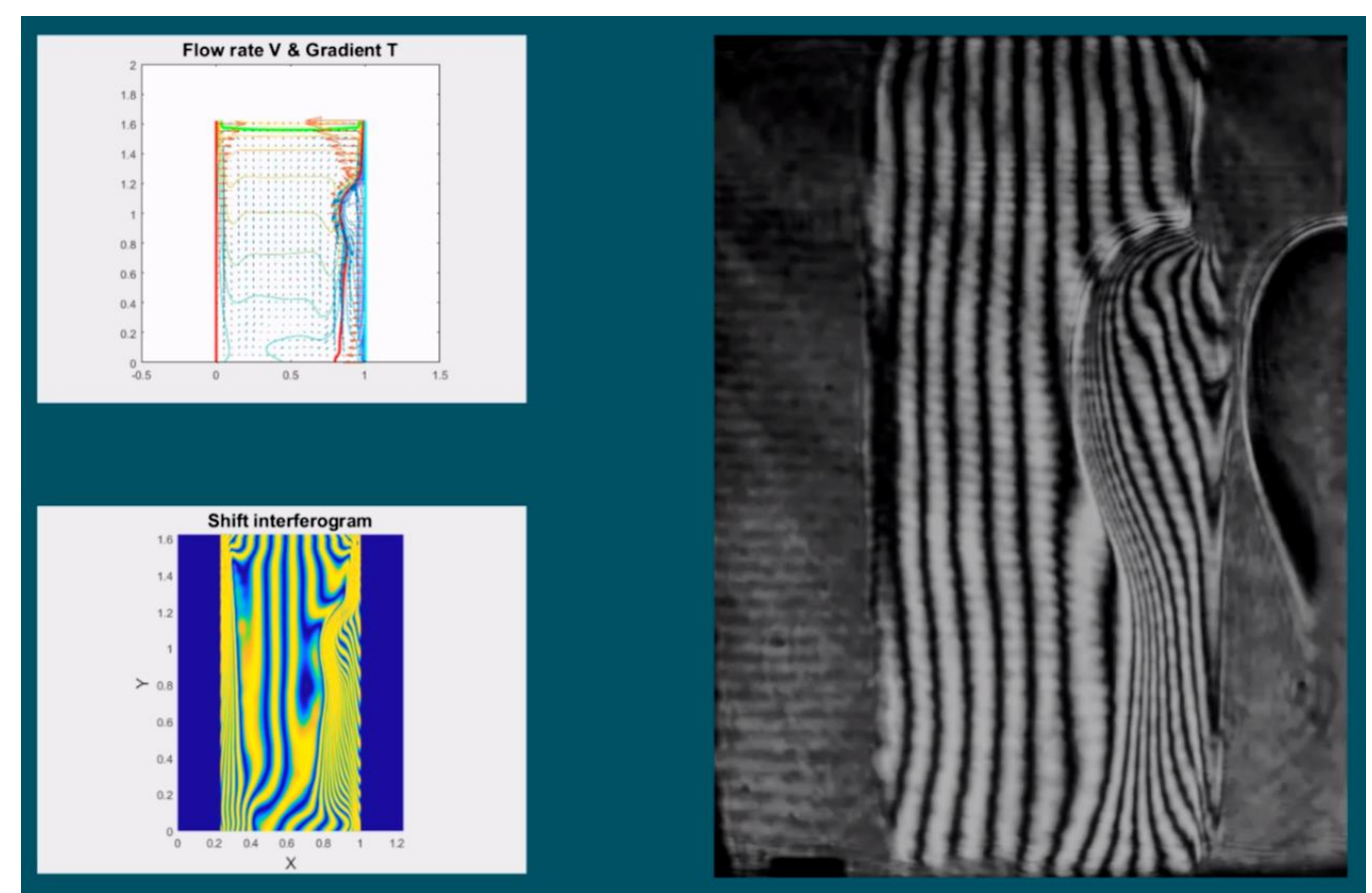

Fig. 6. Reconstruction of shear interferograms of the optical density field.

At the top left a simulation of evolution of the fields of isotherms, temperature gradients and velocity fields is shown, at the bottom left - shear interferograms reconstructed from the numerically obtained field of isotherms. On the right, the figure shows an experimentally obtained interference field that displays the structure of the convective flow induced by nonstationary boundary conditions. The film illustrates the qualitative agreement between the results of numerical simulation and experiment.

\section{Conclusion}

Using the methods of Hilbert-optics, shearing interferometry and numerical simulations, we studied the evolution of convective structures induced by unsteady boundary conditions in the vertical layer of water bounded by flat heat exchange surfaces, in conditions of monotonic cooling. The problem of reconstructing Hilbert images and shear interferograms of the fields of phase optical density from the numerically obtained evolution of isothermal fields and velocity is solved. Studies of convective flows induced by unsteady boundary conditions are relevant for solving various fundamental and applied problems, an example of which is the development of new technologies for crystal growth, understanding of convective processes in the water column in the study of the Arctic and Antarctic regions of the world ocean.

The work was performed within the state task of IT SB RAS (State. reg. AAA-AH17117030310010-9 and AAA-A17-117022850021-3). 


\section{References}

1. Lappa M. Thermal Collection: Patterns, Evolution and Stability. Chichester: John Wiley Sons. 2010.

2. Yu. N. Dubnishchev, V. A. Arbuzov, E. V. Arbuzov, V. S. Berdnikov, S. A. Kislitsyn, O. S. Melekhina. Optical diagnostics of convective structures induced by unsteady boundary conditions in a vertical water layer. Scientific visualization. 2018. Vol. 10, № 4. P. 134-144. DOI: 10.26583/sv.10.4.10.

3. Soloveychik Yu. G., Royak M. E., Persova M. G. Finite element Method for solving scalar and vector problems. Novosibirsk: NGTU. 2007. 896 P.

4. Skvortsov, A.V. Delaunay Triangulation and its application. Tomsk: Tomsk state University. 2002. $128 \mathrm{P}$. 1978.

5. Ivanov A. Introduction to Oceanography. Publishing House "Mir", Moscow.

6. Tables of standard reference data: Water. Density at atmospheric pressure and temperatures from $\mathrm{O}^{\circ} \mathrm{C}$ to $1000^{\circ} \mathrm{C}$. gssd 2-77. Publishing standards. Moscow. One thousand nine hundred seventy eight.

7. Arbuzov V.A., Arbuzov E.V., Berdnikov V.C., Dubnishchev Yu.N., Melekhina O.S. Dynamics of the Crystallization Front Induced by the Temperature Gradient at the Upped Boundary of a Horizontal Layer of a Fluid // Optoelectronics, Instrumentations and Data Processing. 2017. V. 53, No 2. P. 131-135. DOI: 10.3103/S87566990170220042. 